\title{
Finite Size Corrections in the Two-Level BCS Model
}

\author{
A. Ciechan and K.I. Wysokiński \\ Institute of Physics and Nanotechnology Centre \\ M. Curie-Skłodowska University \\ Radziszewskiego 10, 20-031 Lublin, Poland
}

\begin{abstract}
Superconducting properties of small metallic grains modelled by highly degenerate two-level spectrum have been studied. We have solved numerically Richardson's exact equations for the system of $2 N$ electrons in two levels. Characterising the size of the grain by the level degeneracy we study the finite size corrections to the thermodynamic limit of the ground and lowest excited state energy. The interparticle distance $\propto N^{-1 / 3}$ seems to be the expansion parameter. The obtained results have been compared with those of other authors.
\end{abstract}

PACS numbers: 61.46.+w, 73.22.Gk, 74.90.+n

\section{Introduction}

The properties of materials at nanoscale differ from their equivalents in the bulk. This is due to the discrete energy spectrum, parity effects, and large surface to volume ratio. The characteristics depend on the size of the grain which may be quantified by the total number of particles in it [1-3].

We consider a small system with two highly degenerate energy levels close to the Fermi energy with $2 N$ electrons interacting by the weak attractive potential. We model it by the following Hamiltonian:

$$
H=\sum_{f, \sigma= \pm} \epsilon_{f} c_{f \sigma}^{+} c_{f \sigma}-g \sum_{f, f^{\prime} \in S} c_{f+}^{+} c_{f-}^{+} c_{f^{\prime}-} c_{f^{\prime}+},
$$

where $(f \sigma)$ denotes the single particle quantum numbers, $\epsilon_{f}=\epsilon_{1}, \epsilon_{2}$ - the single electron energy levels with $\epsilon_{2}-\epsilon_{1}=h$ and $g$ is the positive coupling constant, $c_{f \sigma}^{+}, c_{f \sigma}$ are fermion creation and annihilation operators. The degeneracy of each level is assumed to be the same and equal $N$.

\section{Theory and results}

Richardson and Sherman [4] have shown that eigenproblem defined by this Hamiltonian can be solved exactly. For $\epsilon_{1(2)}=\mp \epsilon$ the solution reduces to $(N+1)$ 
algebraic equations

$$
\left(\omega_{\mu}-E\right) \varphi(\mu)-A_{\mu} \varphi(\mu+2)-B_{\mu} \varphi(\mu-2)=0,
$$

where $E$ is the total energy of the system, $\varphi(\mu)$ - its eigenfunction,

$$
\begin{aligned}
& \omega_{\mu}=2 \mu \epsilon-\frac{1}{2} g\left[N\left(\Omega_{1}+\Omega_{2}-N+2\right)+\mu\left(\Omega_{2}-\Omega_{1}-\mu\right)\right], \\
& A_{\mu}=\frac{1}{4} g\left[N\left(2 \Omega_{2}-N\right)+\mu\left(\mu-2 \Omega_{2}\right)\right], \\
& B_{\mu}=\frac{1}{4} g\left[N\left(2 \Omega_{1}-N\right)+\mu\left(\mu+2 \Omega_{1}\right)\right] .
\end{aligned}
$$

The argument $\mu=N_{2}-N_{1}(\mu=-N,-N+2+\cdots, N)$ of the function is the difference between the number of pairs in upper and lower energy level. Solving these equations one obtains $(N+1)$ roots for the energy $E$ and the corresponding wave functions $\varphi(\mu)$. The lowest of the roots is the ground state energy of the system, the others are excited states. There exist also pair-breaking excitations, which are obtained by breaking the $N^{\prime}$ pairs and placing non-interacting $2 N^{\prime}$ electrons on the two levels [3].

For the numerical purposes we assume no unpaired electrons in the ground state of (1), replace [5,6] $g$ with $g / 2 N$ and take $\epsilon=h / 2$.

We focus first on the ground state energy $E_{\mathrm{GS}}$. Richardson [5] showed that in the regime $h / g<1$

$$
e=\frac{E_{\mathrm{GS}}}{2 N}=-\frac{1}{4 g}\left(h^{2}+g^{2}\right)+\frac{1}{4 N}(\Delta-2 g)-\frac{1}{16 N^{2}} \frac{g(3 g-\Delta)(g-\Delta)}{\Delta^{2}}+\cdots,(6)
$$

where the BCS gap $\Delta=\sqrt{g^{2}-h^{2}}$.

The first term in (6) is the thermodynamic limit of the ground state energy. It agrees with the BCS value. Other terms are the first and the second-order corrections, respectively.

The regime $h / g>1$ was studied in a recent paper [6] where continuous unitary transformation technique has been used to find the function $e(N)$ of the same model. For $g=1, h>1$ it reads

$$
\begin{aligned}
e= & -\frac{h}{2}+\frac{1}{2 N}(-h+\sqrt{h(h-1)}) \\
& +\frac{1}{4 N^{2}}\left(-\frac{2 h-1}{2(h-1)}-\frac{h}{\sqrt{h(h-1)}}\right)+\cdots
\end{aligned}
$$

At the critical point $h=1$ they have found

$$
e(N)=-\frac{1}{2}-\frac{1}{2 N}+\frac{1}{(2 N)^{4 / 3}}+\cdots
$$

It turns out that the solution of Eqs. $(2-5)$ covers both regimes. In Fig. 1a we show $e(N)$ for $h / g=1$ and the function $e(h / g) / g$ for fixed $N$ in Fig. 1b. It is continuous at the point $h=1(g=1)$ and coincides with Eq. (6) in the regime $h<1$ and Eqs. $(7,8)$ for $h>1$. The thermodynamic limit of the ground state is $e(N \rightarrow \infty)=-\left(h^{2}+g^{2}\right) / 4 g$ for $h / g<1$ and $e(N \rightarrow \infty)=-h / 2$ for $h \geq 1, g=1$. 

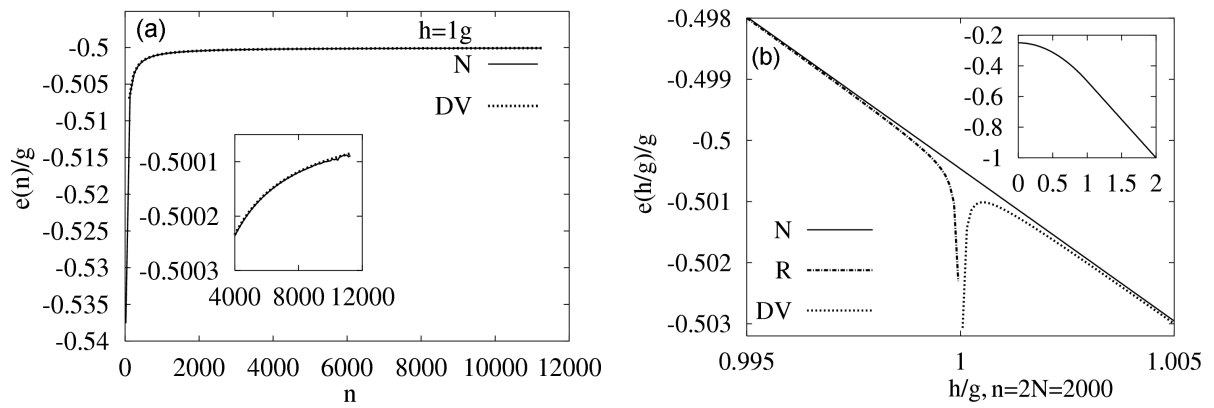

Fig. 1. The ground state energy per electron $e=E_{\mathrm{GS}} / 2 N$ in units of $g$ as a function of the number of electrons $n=2 N$ for $h=g=1$ (a) and $h / g$ for $n=2000$ (b). Our results (solid lines) are compared with those of [5] (dashed line $-R$ ) and [6] (dotted lines $-D V)$. The insets show respective data on an expanded scales.

Now, we consider the lowest excited states $E_{\mathrm{I}}$, which preserve the total number of pairs $N$. The difference $E_{\mathrm{ex}}^{(1)}=E_{\mathrm{I}}-E_{\mathrm{GS}}$ is proportional to the BCS order parameter $\Delta$ in the thermodynamic limit. Richardson [5] has found in the regime $h / g<1, h=1$

$$
E_{\mathrm{ex}}^{(1)}=\Delta-\frac{g}{N \Delta^{2}}\left(1+2 g^{2}-2 g \Delta\right)+\cdots .
$$

On the other hand, in the regime $h \geq 1, g=1$ it has been found [6] that

$$
E_{\mathrm{ex}}^{(1)}=\frac{\alpha}{(2 N)^{1 / 3}}+\cdots, \quad \text { for } \quad h=1
$$

and

$$
E_{\mathrm{ex}}^{(1)}=2 \sqrt{h(h-1)}+\frac{1}{2 N}\left(\frac{4 h-1}{h-1}-2 \frac{h}{\sqrt{h(h-1)}}\right)+\cdots, \quad \text { for } h>1 .
$$

These approximate results are compared with our numerical calculations in Fig. 2. It is worth noting that the excitation energies have different behaviors in

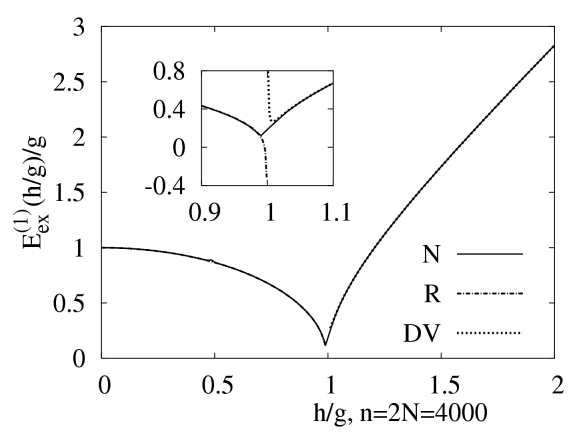

Fig. 2. Dependence of the first excitation energy on $h / g$ for fixed $N=2000$. The inset shows the comparison with analytical results near the point $g=h=1$. 
two regimes of interest $h / g<1$ and $h / g>1$. The position of the kink slightly depends on $n$ and moves towards the point $g / h=1$ for $n \rightarrow \infty$.

\section{Conclusions}

We have numerically solved Richardson's equations for a two-level BCS model with even number of electrons $n=2 N$ and found the ground state $E_{\mathrm{GS}}$ and excitation $E_{\mathrm{ex}}^{(1)}$ energies. There are two regimes of the model $h / g<1$ and $h / g>1$ marked by the kink of the excitation energy. The calculated ground state energy is continuous at this point. The approximate expressions obtained analytically by Richardson [5] and Dusuel, Vidal [6] by different methods agree remarkably well with our exact results in appropriate limits. The detailed dependence of states and excited energies on $N$ will be a subject of future studies.

\section{Acknowledgments}

This work has been partially supported by the State Committee for Scientific Research grant No. 2P03B 06225.

\section{References}

[1] J. von Delft, D.C. Ralph, Phys. Rep. 345, 61 (2001).

[2] K.A. Matveev, A.J. Larkin, Phys. Rev. Lett. 78, 3749 (1997).

[3] E.A. Yuzbashyan, A.A. Baytin, B.L. Altshuler, Phys. Rev. B 71, 094505 (2005).

[4] R.W. Richardson, N. Sherman, Nucl. Phys. 52, 221 (1964).

[5] R.W. Richardson, J. Math. Phys. 6, 1034 (1965).

[6] S. Dusuel, J. Vidal, Phys. Rev. A 71, 060304 (2005). 